\title{
Secretoneurin: twice is not enough
}

\section{By Tim Fulmer, Staff Writer}

The graveyard of stroke compounds has been filled by both thrombolytics, which restore blood flow, and neuroprotectants, which protect brain tissue from infarction. Recently, researchers reported in The Journal of Clinical Investigation that the endogenous peptide secretoneurin has two potential roles in stroke-neuroprotection and recruitment of stem cells to the brain to potentially help replace damaged tissue. ${ }^{1}$

Despite these intriguing dual roles, clinicians and biotech companies focused on stroke therapies are not holding out much hope for secretoneurin, for reasons specific to the indication and the target itself.

In the U.S., the only drug approved to treat ischemic stroke is the thrombolytic Activase alteplase, a tissue plasminogen activator (tPA) from Genentech Inc.

Last year saw a setback on the thrombolytic side, as Paion AG's desmoteplase, a genetically engineered salivary plasminogen activator from the vampire bat Desmodus rotundus, failed to show a significant improvement over placebo in the Phase III DIAS-2 trial to treat acute ischemic stroke. ${ }^{2}$

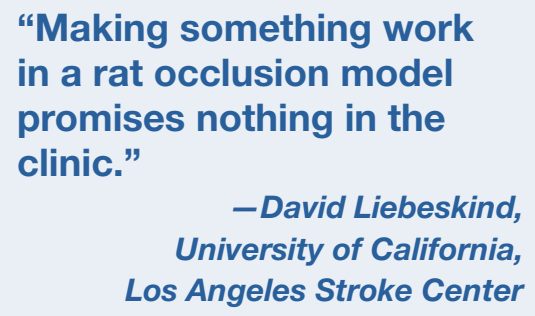

higher than those in controls as measured using an ELISA. Subsequent MRI studies revealed lower infarct volume in rats receiving intravenous secretoneurin than in controls at 7-28 days after induction of cerebral ischemia.

Preliminary immunostaining data in the paper also suggest that secretoneurin could have the ability to recruit stem cells from peripheral circulation and mobilize them to target ischemic sites in the brain. If so, the stem cells might engraft and differentiate into neuronal and glial cells.

The paper's corresponding authors did not return requests for comments on how they believed secretoneurin compared with other neuroprotectants in preclinical and clinical development. Nor did they disclose next steps to SciBX.

Armin Schneider, VP of molecular neurology at Sygnis, told SciBX that he was intrigued by secretoneurin's multimodal potential to treat acute ischemic stroke and promote neurogenesis. However, clinicians and another company in the stroke space, NeuroProtect Inc., were more skeptical.

David Liebeskind, associate neurology director at the University of California, Los Angeles Stroke Center, questioned the strategy of identifying endogenously upregulated proteins during ischemia as potential stroke therapeutics. "The fact that secretoneurin's upregulation is correlated with cerebral ischemia may imply that its efforts are already spent," he said. He questioned whether pushing the concentration of the neuropeptide even higher would yield a better response.

Geoffrey Donnan, professor of neurology at the University of Melbourne and director of the National Stroke Research Institute in Australia, added that data from rodent stroke

There are no approved neuroprotectants for the indication, and the highest-profile compound to date-NXY-059 from Renovis Inc. - failed to significantly lower stroke-related disability in the Phase III SAINT II trial in October 2006. ${ }^{3}$

Instead of pursuing synthetic small molecules, a different strategy has been to identify endogenous proteins that are upregulated in stroke. On this front, Sygnis Pharma AG recently reported data for its G-CSF AX200 to treat acute ischemic stroke. ${ }^{4}$

In the Phase IIa AXIS trial in 44 patients, the compound met the two primary endpoints of similar rates of serious adverse events and thromboembolic complications compared to placebo. Also, a post hoc analysis revealed that AX200 improved infarcts with initial diffusion deficits $>16 \mathrm{~cm}^{3}$ (those in patients with more severe stroke) in the study's per protocol population. Sygnis said it plans to begin a Phase IIb trial this year in about 300 ischemic stroke patients.

Along similar lines, the JCI paper, by a team of Chinese researchers from Academica Sinica and China Medical University and Hospital, illustrates the neuroprotective properties of secretoneurin, a neuropeptide derived from secretogranin II.

Secretoneurin levels in brain samples from rats undergoing cerebral ischemia and in the sera of acute stroke patients were significantly models are not predictive of neuroprotection in stroke patients.

Liebeskind agreed, adding that "making something work in a rat occlusion model promises nothing in the clinic."

According to Donnan, one potential solution to the dearth of animal models is to study in vitro efficacy in human tissue samples-in particular, fresh brain slices from biopsy specimens. He noted, however, that ethical hurdles could prevent use of the approach in many countries.

In clinical trials, said Donnan, imaging techniques such as PET should be used to demonstrate that a neuroprotectant crosses the blood-brain barrier and reaches the ischemic penumbra in sufficient concentration.

Roger Simon, chairman and director of the Robert S. Dow Neurobiology Laboratories and a cofounding scientist at NeuroProtect, said that for secretoneurin to have a chance of making it into the clinic, the researchers' next steps should include determining the compound's dose-response curve, its time window of action and its ability to cross the blood-brain barrier. Researchers also need to show efficacy in an animal model of stroke, preferably in primates, Simon said.

The company has two lead compounds in preclinical development. One, NPI-503, targets toll-like receptors (TLRs) and would be given 


\section{TARGETS \& MECHANISMS}

prophylactically to patients at greatest risk of stroke, for example following a transient ischemic attack. The compound is designed to act as a noninjurious, preconditioning stimulus to trigger adaptive events in brain cells that increase resistance to subsequent ischemic injury, a state known as ischemic tolerance. ${ }^{5}$

The other compound, NPI-505, is an inhibitor of acid-sensing ion channel-1 (ASIC1) that blocks calcium toxicity and is designed to protect against tissue damage resulting from acute ischemic stroke.

To avoid the limitations and pitfalls associated with using a single preclinical stroke model, Simon said NeuroProtect is taking a multitiered approach to discovery and development. Leads are progressed through a series of in vitro studies, rat models and primate models.

\section{REFERENCES}

1. Shyu, W.-C. et al. J. Clin. Invest.; published online Dec. 13, 2007; doi: $10.1172 / \mathrm{JCl} 32723$

Contact: Hung Li, Institute of Molecular Biology, Academica Sinica, Nankang, Taipei, China e-mail: hungli@ccvax.sinica.edu.tw

Contact: Woei-Cherng Shyu, Center for Neuropsychiatry, China Medical University and Hospital, Taichung, Taiwan, China

e-mail: shyu9423@yahoo.com.tw

2. Ku, A. BioCentury 15(25), 14-25; June 4, 2007

3. Edelson, S. BioCentury 14(48), 7-8; Oct. 30, 2006

4. Ku, A. BioCentury 15(35), 14; Aug. 6, 2007

5. Gidday, J.M. Nat. Rev. Neurosci. 7, 437-448 (2006)

\section{COMPANIES AND INSTITUTES MENTIONED}

Academica Sinica, Taipei, China

China Medical University and Hospital, Taichung, China Genentech Inc. (NYSE:DNA), South San Francisco, Calif.

National Stroke Research Institute, Melbourne, Australia

NeuroProtect Inc., Tigard, Ore.

Paion AG (FSE:PA8), Aachen, Germany

Robert S. Dow Neurobiology Laboratories, Portland, Ore.

Renovis Inc., South San Francisco, Calif.

Sygnis Pharma AG (FSE:LIO), Heidelberg, Germany

University of California, Los Angeles Stroke Center, Los Angeles, Calif.

University of Melbourne, Melbourne, Australia 